\title{
Analysis of the Relationship Between form and Qi in Architectural Space
}

\author{
Ni Jinwei ${ }^{1}$, Yang Jiqing ${ }^{1,}$, , Li Wancai ${ }^{1}$, Lu Zhiyuan ${ }^{2}$ \\ ${ }^{1}$ College of Architectural Engineering, Yunnan Agricultural University, Kunming, China \\ ${ }^{2}$ College of Landscape and Horticulture, Yunnan Agricultural University, Kunming, China \\ Email address: \\ 497447133@qq.com (Ni Jinwei), 33478983@qq.com (Yang Jiqing),419373164@qq.com (Li Wancai), 1494652196@qq.com (Lu Zhiyuan) \\ ${ }^{*}$ Corresponding author
}

To cite this article:

Ni Jinwei, Yang Jiqing, Li Wancai, Lu Zhiyuan. Analysis of the Relationship Between form and Qi in Architectural Space. International Journal of Architecture, Arts and Applications. Vol. 6, No. 1, 2020, pp. 12-16. doi: 10.11648/j.ijaaa.20200601.12

Received: November 16, 2019; Accepted: February 17, 2020; Published: April 8, 2020

\begin{abstract}
This paper discusses the concept and essence of "Qi" in Chinese traditional culture. It is considered that "Qi" is the source of all things, and "Qi" in architectural space is the unity of material, energy and information flow flowing and changing in architectural space. It is the basic condition and operation mechanism of life activities and events. Three main aspects of the influence of "form" on "Qi" of architectural space are put forward: air flow, sound field, sunlight and other radiation. This paper analyzes on the relationship between "Qi" and "form" in architectural space, and expounds the influence mechanism of space form on "Qi". The "forms" of space should be conducive to the harmony of Yin Qi and Yang Qi. Different "form" have different effects on the distribution of sound field energy and information in the space, and this difference can have a direct and significant impact on the user's personal feelings, that is, different "shapes" form different "Qi", so as to have a significant impact on the actual effect of the building space. The appropriate "forms" of the building can obtain the right amount of sunlight (visible light, infrared, ultraviolet), shield harmful rays and other high-speed flying objects. The space form follows the principle of "lucky Qi goes curve", which can play the role of shielding through layers of barriers. The architectural space full of "Qi" is the ideal living environment we pursue. The "forms" of architectural space can have an important influence on the generation of "Qi". Only when the building adopts the appropriate "forms", can it get the space that is really suitable for users, and form "Qi" in the space, so as to facilitate the harmonious coexistence of people and environment. The ultimate goal of architectural design and construction practice is to find and build places that can produce "Qi".
\end{abstract}

Keywords: Architectural Space, Form, Qi, Building Atmosphere, Phenomenology

\section{Introduction}

\subsection{The Origin of the Concept of "Qi"}

"Qi" is a unique concept in Chinese traditional culture. It is believed that everything is produced by Qi. The concept of Qi is perfectly integrated into the theory of traditional Chinese medicine. Qi originated from the ancient Chinese philosopher Lao Tzu's thought of cosmic evolution, as Lao Tzu said in Chapter 42 of Tao Te Jing: "Tao gives birth to one, one gives birth to two, two gives birth to three, three gives birth to all things." [1] Here, "one" is Taiji, "two" is "Yin-Yang", and the"three"is "Yin-Yang harmony". It is believed that "Promordial Qi" is a chaotic entity before the separation of heaven and earth, and "Qi" condenses into tangible things and runs through them both inside and outside. [2]

According to George Lemmett, a Belgian clergyman and physicist, the Big Bang theory on the origin of the modern universe was first proposed. The universe evolved from an extremely dense and high temperature primitive state before a limited period of time in the past. After that, everything in the universe was formed by rapid expansion, cooling and condensation. In ancient Chinese theory of Qi, the chaotic unity of Qi is similar to the state of the universe before the Big Bang. After a long evolutionary process, a part of our world "Qi" has evolved into a specific form, but the rest of the world still maintains the state of "Qi", which can be transformed into each other when conditions change.

Limited to the level of ancient people's understanding of the 
material world, Chinese ancient philosophers classified the visible and touchable matter as "form" and the material that could not be observed directly as "Qi". As we know today, the particles, molecules and atoms that make up matter; air, light, heat, radiation rays, biological enzymes and excitation. Nutrients, genes, etc.

"Qi" is the source of all things, including substances, energy and information (such as genes) that germinate new things (including new life and new cells), and it has certain function information function under certain conditions, including its operation rules. More descriptions of "Qi" are related to life, such as birth of new life, seed germination, cell division and regeneration, growth and decay of all things, etc. The "Qi" conducive to life germination and growth of all things is called "lucky Qi". The harmful gases such as sulphur dioxide and carbon dioxide become "bad Qi". The harmful rays to human body are called "evil Qi". Traditional Chinese medicine applies the concept of "Qi" to explain the internal operation of the human body. It is believed that "Qi" is a substance with life information energy. [3]

\subsection{Discussion on the Essence of $Q i$}

So far, scholars at home and abroad have not obtained a satisfactory result for all. Idealists believe that it is a derivation of objective spirit, while materialists believe that it is an element that constitutes the origin of the world. But they generally believe that Qi is ubiquitous, that Qi constitutes everything, and that Qi is constantly moving and changing [4]. Others believe that "Qi" is a pure mode of thinking, which originates from the theory of empty constant motion. [5]

Some scholars have made extensive research on the essence of "Qi" by using modern physics. A more authoritative viewpoint holds that the essence of "Qi" is ultrafine particles and their fields. Some scholars believe that Qi is the most basic substance constituting the universe, Qi is the most basic substance constituting the human body, and Qi is the most basic substance maintaining human life activities. In 1996, Deng Yu and others put forward that Qi in TCM (Traditional Chinese Medicine) theory is a pluralistic unity and generalized wave of "information-energy-matter-space-time". [6, 7, 8]

\section{3. "Qi" in Chinese Geomantic Omen Theory}

Ancient Chinese geomantic omen theory is a philosophy that studies the law of environment and universe. The early geomantic omen was mainly related to the methods and principles of site selection, location and construction of palaces, houses, villages and cemeteries. The theory holds that "form" and "Qi" are two important aspects of analyzing the architectural space environment, and the quality of "Qi" is the ultimate standard to measure the quality of architectural space. Guo Pu (A. D. 276-324), an ancient Chinese scholar, has such a statement in the Book of Funeral Classic: "The Qi is tiny of Form, the Form is the show of Qi, the Qi is hidden and difficult to know, and the Form is obvious"."The Qi disperses when the wind blows, and stops when it comes to water."
Because geomantic omen theory believes that "Qi" is the foundation of all things, where there is "Qi" there is hope, where there is hope is "luchy place",

Therefore, the ultimate goal of geomantic omen practice is to find the place where "Qi" can be gathered or generated. For example, Chinese traditional auspicious patterns are widely used in Yunjuan, Wanzi, Fish intestine, Huiwen, etc. These are the Chinese ancients'perceptions and recognition of "Lucky Qi goes curve, Evil Qi goes straight".

Therefore, geomantic omen holds that the place surrounded by mountains and rivers constitutes a specific spatial form, in which Oi must be produced, and it is an ideal place for people to live. Many ancient cities and settlements in China have chosen their sites according to this principle, which has produced good results. Such as the ancient city of Dali in Yunnan Province, China, backed by the snow mountain (Cangshan), facing the lake (Erhai), the climate is pleasant all the year round, rich in products, is an ideal holiday resort.

"Qi" and "Form" are two important aspects of geomantic omen, and they coexist and depend on each other. Form influences the generation and existence of Qi, which is the soul of Form.

\section{The Relation Between "Form" and "Qi" in Architectural Space}

Architecture is an important part of human civilization. People's requirements for architectural space include more spiritual and aesthetic needs from the initial shelter from the wind and rain to various social activities. Our designers today have been trying to make the architectural space fully meet these diverse needs of human activities. From various physical indicators of function, such as temperature, humidity, ventilation, lighting, air composition, to the sound effect, visual perception, atmosphere and cultural characteristics of space, it can be said that there are many complicated factors.

The "Qi" in architectural space is interpreted by the author as the unity of material, energy and information flow flowing and changing in architectural space, which is the basic condition and operation mechanism of life activities and events. Specific content includes light, heat, humidity, wind, sound, gas, magnetic field, and so on. Of course, it also includes information flow transmitted through sound, light, magnetic and other forms.

External factors affecting the internal environment of building space are numerous and complex, such as geographical latitude, sea level, water body, wind direction, building orientation, topography and topography, as well as the composition of the building itself. Most of these factors are related to the specific geographical environment of the building. From the building itself, different materials and thicknesses of building envelopes can affect the temperature environment inside the building; the color and texture of building surface materials can affect people's intuitive feelings, and so on. This paper focuses on discussing the influence of 
"Form" of space on "Qi ". For green buildings with low energy consumption, the influence of "Form" of building space on "Oi" in space is mainly manifested in the following four aspects:

1) Air flow: It affects the heat, humidity, wind speed, gas composition and their distribution field in the interior space of the building.

2) Sound field: It affects the information dissemination and the atmosphere of the space, and its effect directly affects the information exchange and space feeling in the space, as well as the information exchange inside and outside the building.

3) Sunshine: Affects the indoor space heat, visual information exchange, behavior, atmosphere and so on.

4) Magnetic field: The relationship between building orientation and geomagnetic field affects human biological magnetic field.

\subsection{The Relationship Between "Form" and "Airflow"}

In ancient China, due to the limitation of technical conditions, the requirement of building thermal insulation is in contradiction with the requirement of absorbing sunlight and ventilation and heat dissipation. Taking in sunlight and avoiding cold wind to accumulate heat has become one of the most important problems in building space. The "Form" of space should be conducive to the harmony of Yin and Yang. Therefore, there is the saying that "lucky Qi goes curve". If the wind speed is high, the "Qi" will disperse.

In winter, Yin-Qi (cold gas) are abundant and Yang-Qi (warm gas) weak. So we should pay attention to heat preservation and avoid excessive loss of Yang-Qi. In summer, Yang-Qi are abundant and Yi-Qi weak, so we should try our best to introduce Yin-Qi and eliminate Yang-Qi so as to achieve a general balance between Yang and Yin.

The relationship between them is discussed here under the condition of low energy consumption without any air conditioning and ventilation equipment. "Form" affects the distribution of heat, moisture and air composition by affecting the airflow path and the airflow velocity distribution.

The influence of the plane shape of the room on the airflow is mainly reflected in the effect of the air pressure and ventilation, as shown in Figure 1.

In the first case, when the airflow passes through the building directly, a larger wind speed can be obtained. At this time, the heat loss is larger, the dead angle of ventilation is more, and the distribution of air velocity, heat and air composition is not uniform. A sketch of the distribution of airflow velocity is given in the first case of Figure 1 . When the airflow rounds through the building, the airflow velocity decreases, and the difference of various indicators decreases. Figure 1 shows the second and third cases.

The fourth case is the air distribution diagram of traditional Chinese courtyards and living rooms. Because the courtyard is illuminated by the wall, the living room uses a screen to block the air flow forward route, which further reduces the air flow speed and reduces the influence of outdoor cold wind on indoor heat. In winter, it is helpful to keep indoor temperature, so as to get a fresh and warm "auspicious atmosphere". But the disadvantage is that in the hot season, the effect of indoor ventilation and heat dissipation is not ideal, and indoor ventilation is insufficient.

The twists and turns of Lukcy Qi's walking route is the principle of space disposal that should be observed in traditional Chinese residential buildings.

As shown in Figure 2, in the "Beijing courtyard" this kind of dwelling, pay attention to ventilation, gas can not directly rush into the hall or bedroom, otherwise unlucky. In order to avoid the air rush, one method is that the gate must be dislocated with the two doors, and the other method is to put a wall in front of the door of the house. In order to keep "airflow smooth", the wall can not be closed. Therefore, it has formed the unique architectural form of "Zhaobi"__ a screen wall facing the gate of a house, in Chinese traditional architecture.

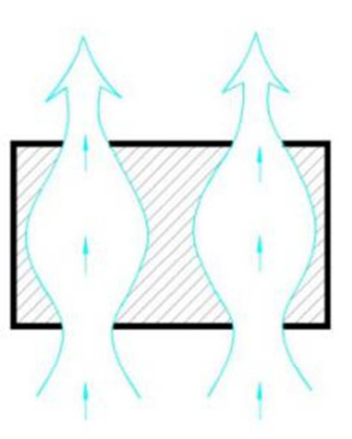

1) Air flows directly through the room

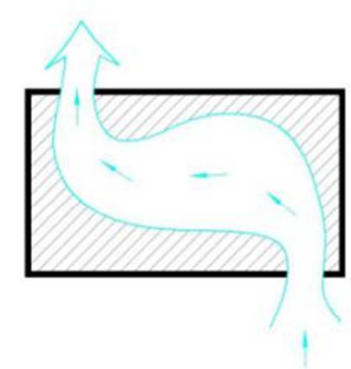

2) Turn the air through the room

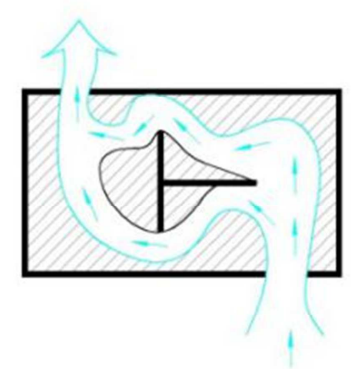

3) Air flows through more complex room

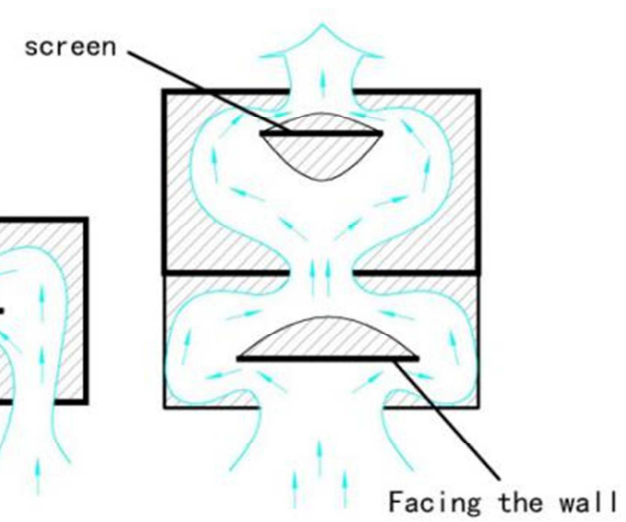

4) Air flow through the traditional Chinese parlor

Note: The shaded part indicates that the air flow is weak.

Figure 1. Distribution of airflow though different types of rooms. 


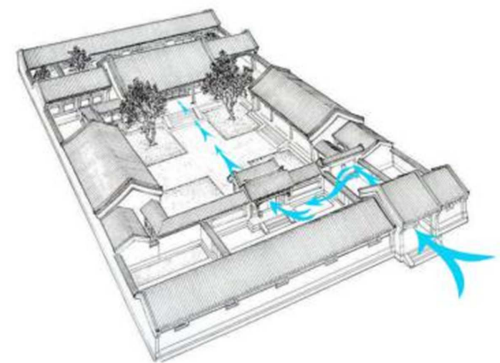

Figure 2. Ventilation Path Analysis of Beijing Siheyuan.

The profile shape of the building space will also have an impact on the ventilation effect, at which time the ventilation height (hot pressure) has the most significant impact. In Chinese traditional geomantic omen theory, this influence has not been paid attention to.

\subsection{The Influence of "Form" of Architectural Space on "Sound Field"}

The sound field in building space contains abundant energy and information, and its quality, change and distribution have an important influence on the formation of "Qi" in space. Different "Forms" have different effects on the distribution of sound energy and information in space, and this difference has a direct and significant impact on the user's personal feelings, that is, different "Forms" can form different "sound fields".

Sound intensity, audio frequency and reverberation time can be used to describe the characteristics of sound in building space. The reflection, transmission and absorption of sound waves by the shape and surface of space are the main factors affecting the characteristics of sound transmission. Sound image and visual characteristics of space, such as shape (shape, scale, location, direction), surface features (material, color, texture, etc.), give the overall impression of the formation of the overall atmosphere of space.

When the interior space of the building is regarded as a large musical instrument, many rooms with different shapes and uses form different vocal chambers with different characteristics, which is equivalent to forming a rich and colorful band. When people travel in different spaces, they constantly hear different "instruments" playing. In fact, the performer is exactly the person who is active in the ensemble. Of course, the wind and underwater sound of nature will also join the ensemble. All these voices form an atmosphere of different characteristics.

The different shape of each cavity in the architectural space can get different performance effects of "musical instruments". Different ways of communication in architectural space require appropriate atmosphere, i. e. different dimensions and combinations of space. For example, in three different types of space: auditorium, banquet hall and study, the required atmosphere is different, and the requirements for space size, openness, sound intensity, reverberation time and sound quality are also different. The right sound atmosphere is where the "atmosphere" lies, because it contains the right energy and information. Therefore, to experience a building, you need to go through it personally, to "play", and the "Qi" you experience is the soul of the building.

According to the sharing range and transmission mode of sound energy information, the basic types of architectural space "shape" can be divided into seven categories: [10]

1) Diffusion type: Sound diffuses outward in a semi-circular and fan shape in order to convey energy information to more people.

2) Etiquette type: the diffusion of sound has direction limitation.

3) Conductive type: sound is transmitted in a specific direction.

4) Import type: Sound diffusion is direct and interactive.

5) Convergence type: Sound diffusion is inward convergence, there are certain conditions to reduce energy leakage.

6) Cohesive type: the transmission of sound has a strong limit, in order to greatly prevent the leakage of information energy.

7) Private type: the transmission of sound is very limited, only allowed to spread in a very small range, energy information is rarely leaked.
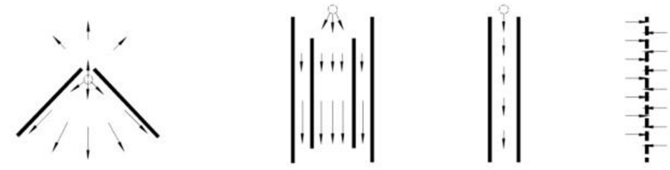

1) Diffusive type

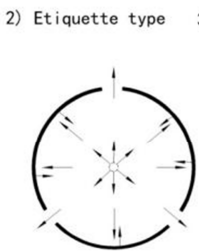

3. Conductive type 4) Interactive type

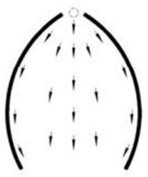

5) Convergence type
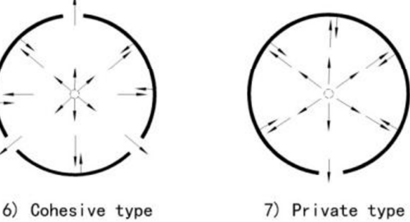

7) Private type

Figure 3. Divides spatial types according to sound propagation characteristics.

The rationality of space can be verified by establishing a complete evaluation technical index system for seven types of space. A good "Form" will produce a good "Qi", and the gathering of "Qi" in space is to gather the sound of resonance consistent with the requirements of the atmosphere, so as to meet people's corresponding physiological and psychological needs.. Different combinations of various spaces can also form the unique personality and charm characteristics of the target building.

\subsection{Relation Between "Form" of Building Space and Sunshine Radiation}

Appropriate "Form" can be used to obtain appropriate amount of sunlight, which can affect the indoor space heat, visual information exchange, behavior, atmosphere and so on.

The building collects sunlight mainly through doors, windows, transparent roofs and lighting wells. Appropriate sunlight intake is conducive to the formation of "Qi" in the 
interior space of the building. Good lighting conditions can be obtained by controlling the orientation of buildings, the form, location and area of doors and windows. The influence of building shape on sunlight absorption is mainly reflected in building orientation, space height, depth, window-to-ground ratio and so on. These have been relatively mature in modern architectural design theory.

In Chinese traditional geomantic omen theory, the influence of architectural orientation on lighting is emphasized. In traditional courtyard buildings, due to the different orientation of the building and the formation of different dignity and inferiority, the building sitting in the north and facing the south is bound to be the easiest to produce "luck Qi". The living room usually uses hollow wood carved doors to increase lighting area, but the bedroom and other room windows are usually very small and insufficient lighting. Considering the safety, privacy requirements of these rooms and the building technical conditions at that time, it is also necessary to do so.

\subsection{The Relationship Between "Form" of Building Space and Magnetic Field}

The strong magnetic field of the earth itself is of great significance to resist the attack of high-speed ions such as solar wind. Without this powerful magnetic force, life on Earth would not exist. The human body itself has biological magnetic field, which is mainly caused by biological current, biological magnetic material and exogenous magnetic material in vivo. When the human biological magnetic field is compatible with the cosmic magnetic field, the normal physiological activities of the internal circulation, tissues and organs of the organism can be maintained, otherwise abnormal reactions will occur and lead to pathological changes.

As far as the environment around the building is concerned, the surroundings surrounded by mountains are conducive to gathering the energy of the magnetic field, shielding harmful rays and high-speed ions, and generating "Luck Qi".

In geomantic omen theory, special attention is paid to the relationship between building orientation and geomagnetism. Commonly used compass is used for field measurement to determine the angle between building orientation and geomagnetism direction. When building orientation is conducive to maintaining harmony between human magnetic field and geomagnetic direction, we can get "Luck Qi".

In the daytime, besides fixing furniture in the same position, people's position is constantly changing. The magnetic field corresponding to the building orientation has less influence on people. But at night, people lie in bed for a long time, accounting for about one third of their lives. The influence of geomagnetic field on human magnetic field will be more significant. Therefore, in addition to the orientation of the building, whether the orientation of the bed conforms to the geomagnetic direction is more important. It is generally believed that when the building is sitting in the north and facing the south, the magnetic field is more advantageous to the human body when the bed is in the North-South direction.

\section{Conclusion}

The building space full of "Qi" is the ideal living environment we pursue. The "shape" of architectural space can have an important impact on the formation of "Qi". Only when the proper shape is adopted in the building, the space suitable for users can be obtained, and the "air" can be formed in the space, which is conducive to the harmonious coexistence of human beings and the environment. As Steven Hall, a contemporary American architect, said, the only building suitable for it is to be found in its place, including the author's ideas and the user's resonance [11]. The ultimate goal of architectural design and construction practice can also be said to be to find and build a place that can produce "Qi", which is also a lofty goal of architectural design.

Sources of pictures in the article: The author draws by himself.

\section{References}

[1] Xin Zhengjun, Laozi [M]. Beijing, Zhonghua Book Company, 2008, 99.

[2] Cheng Yishan, Ancient Chinese Yuanqi Theory [M]. Hubei: Hubei People's Publishing House, 1986, 8.

[3] Wang Huicheng, Guo Xiazhen, On the Essence of Qi, Beijing Biomedical Engineering [J], Vol. 31, No. 6, 2012, pp. 634-636.

[4] Cuijiang, Modern Workplace Fengshui [M]. Shandong Electronic Impression Publishing House, 2008, 6: 21.

[5] Wu Haotian, Wei Cong and others commented on the theoretical research progress of "Qi Essence" in traditional medicine in the past 20 years. Journal of Basic Chinese Medicine [J], Vol. 22, No. 2, 2016, pp281-283.

[6] Deng Yu, Shi Zhongyuan, Shan Baolu and so on, the essence of Qigong's external Qi: "information-energy-matter" unified generalized substance Bo [C], First World Congress on Traditional Life. ASIAMED MAGAZINE, 1996, Oct. SPECIAL PERIOD. 643.

[7] Deng Yu, Zhu Shuoli, Deng Hai, Qi and Generalized Quantum Wave of Traditional Chinese Medicine [J], Journal of Mathematical and Physical Medicine, Vol. 15, No. 4, 2002, pp. 369-371.

[8] Deng Yu, Zhu Shuoli, Xu Peng and Deng Hai, the modern essence of TCM Qi and its quantification: Qiji, Qiyuan [J], number Journal of Science and Medicine, Vol. 11, No. 4, 2003, pp. 346-347.

[9] Peter Zumto, Architectural Atmosphere [M]. Beijing: China Construction Industry Press, 2010.9: 29.

[10] Ni Jinwei, Li Jingyu, Lu Xiang, the influence of architectural space form on Qi [J], Huazhong Architecture, Vol. 18, No. 10, 2015, pp. 27-29.

[11] Liu Quan, Steven Hall and Architectural Phenomenology [J], Chinese and Foreign Architecture, Vol. 16, No. 6, 2007, pp. $38-41$. 